\title{
MOLECULAR DESORPTION BY SYNCHROTRON RADIATION AND STICKING COEFFICIENT AT CRYOGENIC TEMPERATURES FOR $\mathrm{H}_{2}, \mathrm{CH}_{4}, \mathrm{CO} \mathrm{AND} \mathrm{CO}_{2}$
}

\author{
V. Baglin, I.R. Collins, O. Gröbner, C. Grünhagel and B. Jenninger
}

\begin{abstract}
A cryogenic vacuum system has been used to study both the surface pumping of gases and the molecular photodesorption yield from OFE copper in the temperature range from $5 \mathrm{~K}$ to $300 \mathrm{~K}$. The sticking coefficient on a "bare surface" was found to increase with decreasing temperature of the substrate and below $20 \mathrm{~K}$, to be in the range of a few percent for all gas species studied. The molecular photodesorption yield for the "bare surface" was found to decrease with decreasing temperature and below $20 \mathrm{~K}$, after an accumulated photon dose of $3.110^{22}$ photons $\mathrm{m}^{-1}$, to be in the range of $10^{-4}$ molecules photon ${ }^{-1}$ for $\mathrm{H}_{2}$ and $10^{-5}$ molecules photon ${ }^{-1}$ for the other gas species. These measured values are used to predict the evolution of the gas densities in the LHC. Implications to the LHC are discussed.
\end{abstract}

LHC Division

CH - 1211 Geneva 23

Switzerland 


\title{
Molecular desorption by synchrotron radiation and sticking coefficient at cryogenic temperatures for $\mathrm{H}_{2}, \mathrm{CH}_{4}, \mathrm{CO}$ and $\mathrm{CO}_{2}$
}

\author{
V. Baglin, I.R. Collins, O. Gröbner, C. Grünhagel and B. Jenninger \\ CERN, 1211 Geneva 23, Switzerland.
}

\section{Abstract}

A cryogenic vacuum system has been used to study both the surface pumping of gases and the molecular photodesorption yield from OFE copper in the temperature range from $5 \mathrm{~K}$ to $300 \mathrm{~K}$. The sticking coefficient on a 'bare surface' was found to increase with decreasing temperature of the substrate and below $20 \mathrm{~K}$, to be in the range of a few percent for all gas species studied. The molecular photodesorption yield for the 'bare surface' was found to decrease with decreasing temperature and below $20 \mathrm{~K}$, after an accumulated photon dose of $3.110^{22}$ photons $\mathrm{m}^{-1}$, to be in the range of $10^{-4}$ molecules photon ${ }^{-1}$ for $\mathrm{H}_{2}$ and $10^{-5}$ molecules photon ${ }^{-1}$ for the other gas species. These measured values are used to predict the evolution of the gas densities in the LHC. Implications to the LHC are discussed.

\section{Introduction}

In the cryogenic elements of the Large Hadron Collider (LHC), the proton beams will be contained inside a perforated 'beam screen' (BS), operating at a temperature between $5 \mathrm{~K}$ and $20 \mathrm{~K}$. The BS is designed to intercept the heat loads induced by synchrotron radiation (SR), photoelectrons and resistive wall losses to avoid dissipation in the $1.9 \mathrm{~K}$ cold bore (CB) of the superconducting magnets. To provide the required beam lifetime of $\sim 100$ hours, the density of the rest gas must remain below a value determined by the residual gas nuclear scattering of the proton beam (e.g. for $\mathrm{H}_{2} \sim 10^{15}$ molecules. $\mathrm{m}^{-3}$ ). The density is controlled by perforations in the form of 'pumping slots' along the BS such that molecules can reach the CB surface and be cryosorbed [1].

In a cryogenic vacuum system which is exposed to intense SR, the molecular densities are determined by the following effects [2]: 1) 'primary' photodesorption as described by the molecular desorption yield, $\eta$, which constitutes a steady source of 'new' gas, 2) wall pumping characterised by the effective sticking coefficient, $\sigma, 3$ ) pumping via the BS slots $\mathrm{C}, 4$ ) redesorption of condensed gas molecules, $\eta$ ' i.e. the 'recycling' of previously physisorbed molecules by photons and/or by photoelectrons and finally 5) vapour pressure, $n_{e}$ of the condensed species on the cold surfaces.

Due to these effects, the gas densities may increase beyond the specified limit if the correct parameters have not been used for the vacuum system design. Therefore the knowledge of all these parameters over the relevant operating temperature range is of vital importance. A Cold Bore Experiment (COLDEX) has been designed and constructed in collaboration with the NIKHEF Laboratory, with the specific objectives to simulate closely and to validate the operational aspects of the LHC cryogenic vacuum system [3]. This apparatus has been installed at the CERN Electron Positron Accumulator (EPA) using an available SR beam line as the photon source. 


\section{Experimental}

The COLDEX apparatus has been described in detail elsewhere [3]. The essential parts are two concentric tubes, $\sim 2 \mathrm{~m}$ long, with independent temperature control. The outer tube, which represents the $\mathrm{CB}$ tube of the cryo-magnets is screened by the $\mathrm{BS}$ from the direct SR, the inner one, representing as closely as possible the BS of the LHC can be irradiated by SR. The CB, which constitutes the UHV vacuum envelope, is made of $316 \mathrm{LN}$ stainless steel. The BS is made from extruded OFE copper to be representative of the inner $\mathrm{Cu}$ surface of the LHC BS and to ensure temperature uniformity. All materials were cleaned according to CERN standards. The CB has been constructed as a double walled liquid helium container. The BS tube has an inner diameter of $47 \mathrm{~mm}$. It is cooled by circulating gaseous He through two parallel cooling channels located on the outside perimeter. To simulate the conditions of the LHC vacuum system, the BS is perforated with 264 round holes of $4 \mathrm{~mm}$ diameter equally distributed over the full length and representing $1 \%$ of its surface area. Two ports for vacuum gauges are placed in the center of the BS, the upper one for a room temperature (RT) 'chimney' and the lower one for the extractor gauge (EXG). A $1 \mathrm{~mm}$ annular gap was provided to avoid direct thermal contact between the BS ports and the chimney or the cover of the EXG. In this way it is possible to minimise parasitic adsorption on the chimney surfaces and thus to obtain a reliable measurement at RT of the pressure in the test chamber using RGA 3 and BA 4. During a typical run the $\mathrm{CB}$ temperature would be set to $3.1 \mathrm{~K}$ and the $\mathrm{BS}$ temperature would be controlled from 4.5 to $90 \mathrm{~K}$. Alternatively, when the BS is operated above $90 \mathrm{~K}$, the $\mathrm{CB}$ temperature is set to $5 \mathrm{~K}$. All $300 \mathrm{~K}$ data were obtained with the entire apparatus at RT i.e. both $\mathrm{CB}$ and $\mathrm{BS}$.

The beam line for the COLDEX apparatus is shown in Fig. 1. To shorten the irradiation time for a given photon dose, a critical energy of $194 \mathrm{eV}$ was chosen yielding the maximum photon flux from EPA of $3.410^{16}$ photons $\mathrm{m}^{-1} \mathrm{~s}^{-1}$ - about one third of the LHC flux. The SR is collimated by a rectangular aperture $(7.5 \mathrm{~mm}$ horizontally, $11 \mathrm{~mm}$ vertically). The vertical collimation attenuates photons with energies $<4 \mathrm{eV}$ and thus reduces the photon flux (transmission factor of 0.8). In previous RT studies, this reduction of the low energy tail of the spectrum was not considered important since the desorption of the strongly bound species requires a few $\mathrm{eV}$ photons, capable of producing photoelectrons and thus of breaking chemical bonds. This situation is different in a cryogenic system with physisorbed molecules, since molecular desorption by low energy photons with energies of less than $1 \mathrm{eV}$ and even by thermal radiation has been demonstrated to occur [4]. During the setting-up phase COLDEX is aligned according to the theoretical 'straight through' position and the photon beam is centred by adjusting the electron beam orbit. During data taking, COLDEX is moved in the horizontal plane with respect to the 'straight-through' position and the SR irradiates the BS at a grazing incidence with a mean angle of $11 \pm 2.7 \mathrm{mrad}$. In this position, the SR illuminates the BS over $95 \%$ of the total cold length. The COLDEX vacuum system is pumped at each extremity through a conductance of $73.8 \mathrm{l} . \mathrm{s}^{-1}$ for $\mathrm{N}_{2}$. Each of the end pumping systems is a combination of a $200 l . \mathrm{s}^{-1}$ for $\mathrm{N}_{2}$ ion pump and two titanium sublimation pumps of $1000 l . \mathrm{s}^{-1}$ for $\mathrm{H}_{2}$. Six calibrated Bayard-Alpert gauges (BA1 to 6) and one EXG are installed. The gas composition is measured with two quadrupole gas analysers fitted with secondary electron multipliers, RGA2 and RGA3. 


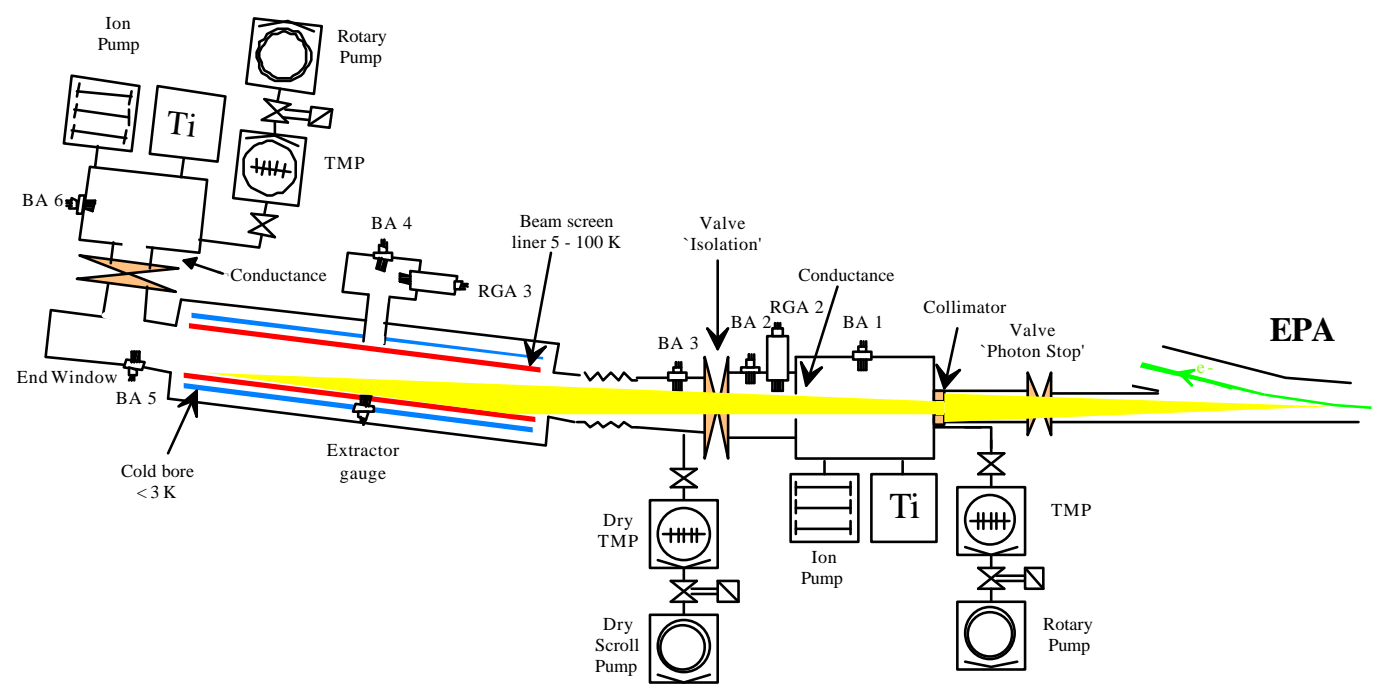

Fig.1. Layout of the beam line with COLDEX.

\section{Principles}

In a cold vacuum system, the sum of the primary photodesorption yield (PPY), $\eta(T)$ and of the recycling photodesorption yield (RPY), $\eta$ '(T) can be expressed by equation (1). This equation involves three unknown quantities, which implicitly depend on the temperature, $T: \eta(T), \eta^{\prime}(T)$ and the sticking coefficient, $\sigma(\mathrm{T})$.

$$
\eta(T)+\eta^{\prime}(T)=\frac{G(T)[\sigma(T) S(T)+C(T)] P \sqrt{\frac{T}{300}}}{\dot{\Gamma}} .
$$

$\mathrm{G}(\mathrm{T})$ is a constant converting from pressure to molecular density, $S(T)$ is the ideal surface pumping speed of the $\mathrm{BS}, \mathrm{C}(\mathrm{T})$ represents the pumping speed by the holes, $\mathrm{P}$ is the partial pressure and $\dot{\Gamma}$ is the total photon flux. The pumping speed $\mathrm{C}(\mathrm{T})$ takes into account the Clausing factor ( $\sim 0.7)$ and the parasitic pumping due to the space between the BS and its surrounding thermal screen $(\sim 0.7 \%$ of the total surface as determined experimentally with an unperforated BS). The square root factor accounts for thermal transpiration. A further condition, which must be fulfilled to use equation (1) is that the vapour pressure can be neglected. This implies either sufficiently low temperatures and/or sufficiently low surface coverages.

If it can be ensured at the beginning and throughout a measurement that the surface coverage remains sufficiently low, the dynamic pressure rise is dominated entirely by the PPY. In this case, the RPY of equation (1) can be neglected, one obtains a simplified expression (2) which involves in addition to known and measured quantities the PPY and the sticking coefficient. In this work, a specific procedure has been adopted to achieve experimental conditions for which this simplified equation can be applied.

$$
\eta(T) \approx \frac{G(T)[\sigma(T) S(T)+C(T)] P \sqrt{\frac{T}{300}}}{\dot{\Gamma}}
$$


For $\mathrm{H}_{2}$, a low surface coverage has been achieved readily by cooling down first the CB and subsequently the BS. This aspect was verified in a control experiment without SR during which the $\mathrm{CB}$ was cooled to $3.4 \mathrm{~K}$ while the BS was maintained initially at $30 \mathrm{~K}$ and only subsequently cooled down to $6.6 \mathrm{~K}$ for 70 hours. From the release of $\mathrm{H}_{2}$ during the following warming up of the $\mathrm{BS}$ an average condensation rate of $610^{11} \mathrm{H}_{2} \mathrm{~cm}^{-2} \mathrm{~h}^{-1}$ could be deduced, equivalent to about $10^{-3}$ monolayer. $h^{-1}$. Since RPY of $\mathrm{H}_{2}$ is 0.1 molecules/photon at one monolayer $[2,5,6,7]$, assuming a linear dependence with coverage, the recycling effect would account for a desorption yield of $10^{-4} \mathrm{H}_{2}$ molecules/photon. This yield being about equal to the PPY one would conclude that the build-up of the condensed layer of $\mathrm{H}_{2}$ on the BS could lead to a modest overestimate of the PPY by up to a factor of two.

The same method of controlled sequential cool down and warm-up has also been used for the other gas species $\mathrm{CH}_{4}, \mathrm{CO}$ and $\mathrm{CO}_{2}$. The upper limits of the condensation rate during a typical COLDEX run which could be measured for these molecular species is $\sim 410^{11}$ molecules $\mathrm{cm}^{-2} \mathrm{~h}^{-1}$. However, since the RPY for these molecules are much smaller than for $\mathrm{H}_{2}[6,7]$, the resulting errors in the measurements of the PPY are proportionally smaller and the uncertainties are in the range of $310^{-7}$ to $310^{-6}$ molecules.photon ${ }^{-1}$.

From equation (2) follows that the pressure in the system depends on both PPY and the sticking coefficient. A practical procedure to evaluate the PPY independently is based on the fact that the dynamic pressure during SR irradiation initially increases but reaches an equilibrium value after a sufficiently long irradiation time [8]. This condition is characterised by the fact that the rate of condensation of molecules on the inner BS surface equals the rate of redesorption due to the recycling effect by the SR. A consequence of this balance is that the surface coverage has reached a constant value. Expressed in different terms, the effective sticking coefficient vanishes since the flow of desorbed gas entering the system is balanced by the flow of gas leaving the system through the holes in the BS. Once this equilibrium pressure $\mathrm{P}_{\mathrm{Eq}}$ has been established, the PPY is obtained.

$$
\eta(T)=\frac{G(T) C(T) P_{E q} \sqrt{\frac{T}{300}}}{\dot{\Gamma}} .
$$

In the case of $\mathrm{H}_{2}$ its large RPY makes it possible to reach the equilibrium within a reasonably short time, i.e. an irradiation time which is readily obtained in an EPA run lasting a few hours only.

For the other gas species, as a consequence of lower RPY than hydrogen, the pressure increase is small, hence difficult to observe, and it may simply take too long to reach the equilibrium. To overcome this difficulty an alternative method to measure the PPY has been used based on the following. Low surface coverage could be achieved by operating the BS above a temperature, $\mathrm{T}_{\mathrm{H}}$ at which the sticking coefficient of a specific molecular species was sufficiently small. With reference to equation (2) this implies that $\sigma$ vanishes. Since physisorbed molecules exhibit well defined activation energies, the recording of the pressure with temperature during a controlled warm-up shows distinct desorption peaks attributed to the respective residual gas species. Due to the exponential dependence of the thermal desorption rate on temperature, the condensed gas is released rapidly within a narrow temperature interval. These desorption peaks depend weakly on the heating rate range ( $<5 \mathrm{~K}$ for $300 \mathrm{meV}$ activation energy and heating rates of $200 \mathrm{mK} / \mathrm{min}$ ). Above $\mathrm{T}_{\mathrm{H}}$, molecules no longer condense on the surface since the sojourn time becomes so short that no significant gas surface phase exists. Therefore, above $\mathrm{T}_{\mathrm{H}}$ the PPY is evaluated using equation (3). For temperatures below $\mathrm{T}_{\mathrm{H}}$, the pessimistic assumption has been made that the PPY remains 
constant and equal to its value at $\mathrm{T}_{\mathrm{H}}$. Below $\mathrm{T}_{\mathrm{H}}$ equation (2) has been used to calculate the sticking coefficient using as input value $\eta\left(\mathrm{T}_{\mathrm{H}}\right)$. The limiting temperatures for the different gas species are 30, 80,65 and $125 \mathrm{~K}$ for $\mathrm{H}_{2}, \mathrm{CH}_{4}, \mathrm{CO}$ and $\mathrm{CO}_{2}$ respectively.

$$
\eta(T)=\left\{\begin{array}{l}
\eta \text { for } T>T_{H} \\
\eta\left(T_{H}\right) \text { for } T \leq T_{H}
\end{array}\right.
$$

\section{Results}

To avoid any significant cleaning of the surface during the measurements the BS was preexposed to a dose of $310^{22}$ photons $\mathrm{m}^{-1}$. Based on the results of a previous experiment at $77 \mathrm{~K}$ a dependence of the PPY with dose $\mathrm{e}^{-1 / 3}$ had been found [5]. Since in all the subsequent measurements the accumulated dose was intentionally limited to $410^{21}$ photons. ${ }^{-1}$, the PPY should have remained constant to within $4 \%$. Fig. 2 shows the PPY as a function of the BS temperature between $5 \mathrm{~K}$ and $300 \mathrm{~K}$. It should be noted that measurements below the limit temperature $\mathrm{T}_{\mathrm{H}}$ are not included in this figure with the exception of $\mathrm{H}_{2}$, for which these data points could be obtained by the independent measurement based on the equilibrium pressure measurement. It can be seen that the PPY decreases with decreasing temperature. The $300 \mathrm{~K}$ data were obtained after an accumulated dose of $3.510^{22}$ photons. $\mathrm{m}^{-1}$. The error bars for each of the measurements derive from the propagation of the individual errors of the partial pressure measurements which have been estimated as $30 \%$ and result from the calculation of the pressure difference with and without photon irradiation. The sequence of measurements was first from $135 \mathrm{~K}$ to $5 \mathrm{~K}$ and subsequently from $100 \mathrm{~K}$ to $300 \mathrm{~K}$.

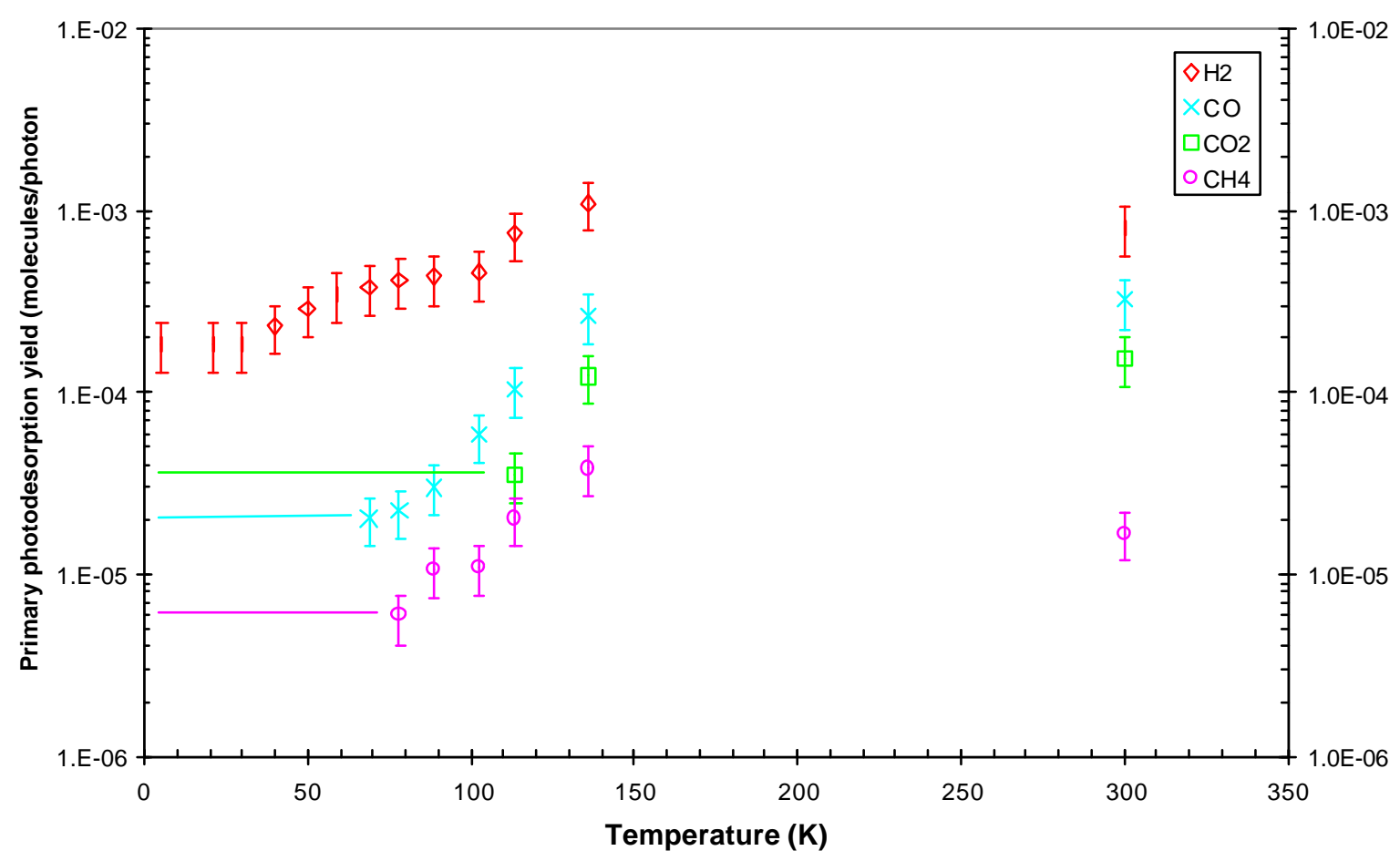

Fig. 2. Primary photodesorption yield for an OFE copper BS irradiated with $194 \mathrm{eV}$ critical energy SR as a function of temperature. The BS had been pre-exposed to an accumulated dose of $310^{22}$ photons $/ \mathrm{m}$. With the exception of $\mathrm{H}_{2}$, measurements below the limiting temperature $\mathrm{T}_{\mathrm{H}}$ have not been included. 
Fig. 3 shows the calculated sticking coefficient from the measured pressure increase using equation (2) and the PPY data in figure 4. The hatched area represents the propagated error derived from the numerical treatment of the data and the partial pressure measurements of each gas species. Below $20 \mathrm{~K}$, the sticking coefficients of all the gases are found to be in the range of a few percent. These values correpond to a 'bare surface' with less than $10^{14}$ physisorbed molecules $/ \mathrm{cm}^{2}$. Comparison with published data of $\mathrm{CO}_{2}$ at $77 \mathrm{~K}$ shows one order of magnitude lower values than given here [9]. Therefore, if the $\mathrm{CO}_{2}$ sticking coefficient was overestimated by one order of magnitude in our experiment, the corresponding PPY of $\mathrm{CO}_{2}$ would proportionally decrease to $10^{-5}$ molecules.photon ${ }^{-1}$.

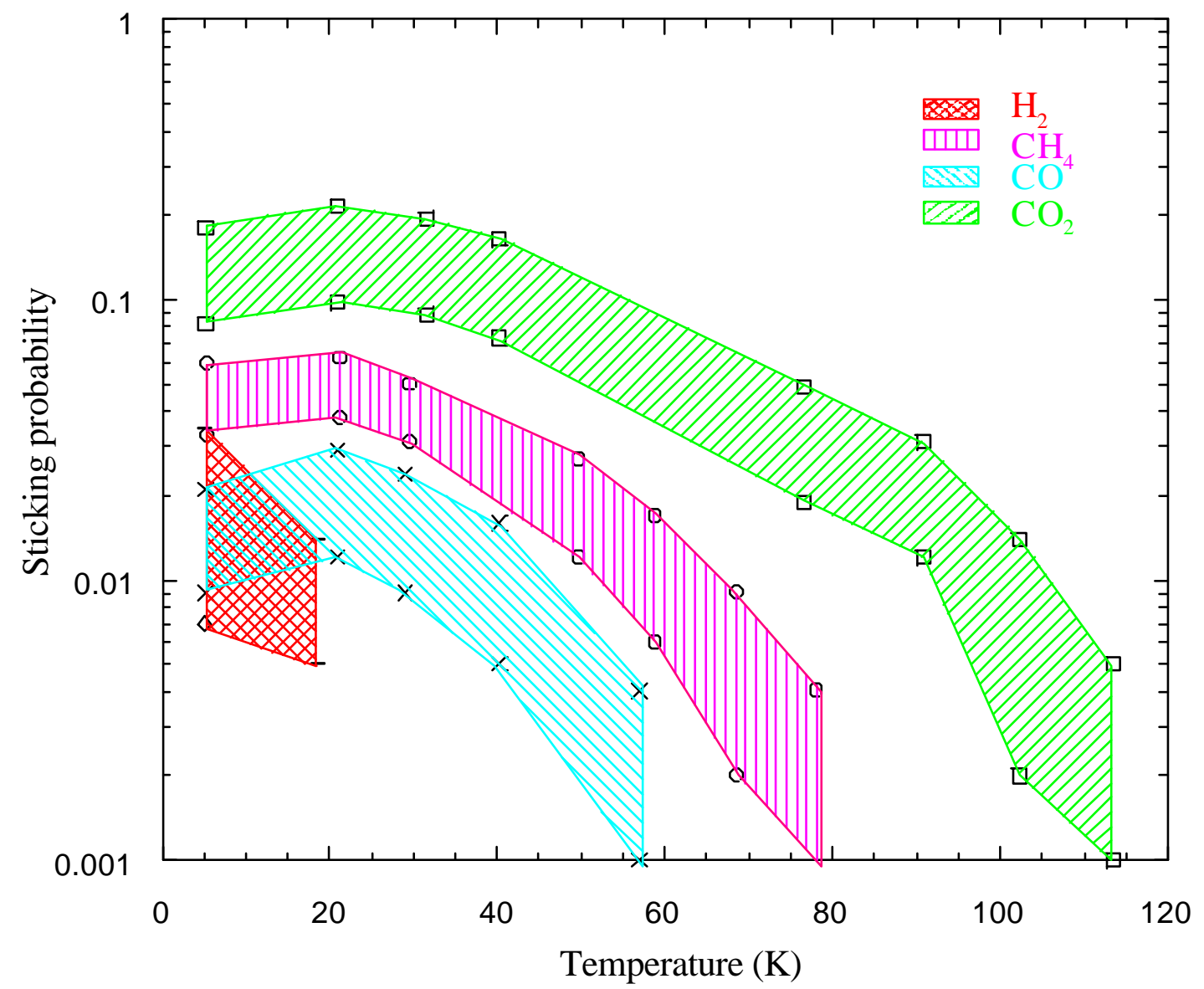

Fig. 3. Sticking coefficients for a 'bare' OFE copper surface below the limiting temperature $T_{H}$.

\section{Implications for the LHC}

To estimate the residual densities and the resulting nuclear scattering beam life time in the LHC, the rather pessimistic assumption has been made that the PPY of all species in the temperature range from $5 \mathrm{~K}$ to $20 \mathrm{~K}$ is equal to its value at the limiting temperature $\mathrm{T}_{\mathrm{H}}$. Table 1 summarises the PPY measured at the indicated temperature T (Fig. 2), the sticking coefficient at $15 \mathrm{~K}$ (Fig. 3), the RPY from Ref [7], the nuclear scattering equivalent gas density with respect to $\mathrm{H}_{2}$, the beam life time corresponding to the individual densities and in the last column, the resulting total beam life time. The nuclear scattering lifetime at $7 \mathrm{TeV}$ of $\mathrm{H}_{2}$ has been calculated using the cross section value $\sigma\left(\mathrm{H}_{2}\right)=$ 
$9510^{-31} \mathrm{~m}^{2}$. The values of the beam life time have been computed for a nominal beam current $(0.56$ A) and for a $\mathrm{BS}$ at the average temperature of $15 \mathrm{~K}$ with $4.4 \%$ pumping holes having a Clausing factor of $\sim 0.5$. The beam life time is dominated by $\mathrm{CO}_{2}$ followed by $\mathrm{CO}$ and with third importance only by $\mathrm{H}_{2}$.

Table 1

Primary photodesorption yield, temperature, recycling yield, relative nuclear scattering cross section at $7 \mathrm{TeV}$ as well as individual and total beam life times.

\begin{tabular}{cccccccc}
\hline Gas & $\begin{array}{c}\eta \\
\text { (molecules/photon) }\end{array}$ & $\begin{array}{c}\mathrm{T} \\
(\mathrm{K})\end{array}$ & $\sigma$ & $\begin{array}{c}\eta^{\prime}[7] \\
\text { (molecules/photon) }\end{array}$ & $\mathrm{n}_{\mathrm{H} 2} / \mathrm{n}_{\text {gas }}$ & $\begin{array}{c}\tau \text { gas } \\
(\mathrm{h})\end{array}$ & $\begin{array}{c}\tau \\
(\mathrm{h})\end{array}$ \\
\hline $\mathrm{H}_{2}$ & $2.010^{-4}$ & $5-20$ & 0.02 & 0.1 & 1 & 1620 & \\
$\mathrm{CH}_{4}$ & $6.010^{-6}$ & 78 & 0.05 & $110^{-4}$ & 6.0 & 3200 & 99 \\
$\mathrm{CO}$ & $2.010^{-5}$ & 69 & 0.02 & $210^{-5}$ & 9.0 & 480 & \\
$\mathrm{CO}_{2}$ & $3.510^{-5}$ & 114 & 0.15 & $110^{-4}$ & 13.9 & 142 & \\
\hline
\end{tabular}

Using the data from Table 1, Fig. 4 shows the evolution of the gas density with running time. Due to its large recycling coefficient, $\mathrm{H}_{\mathbf{L}}$ reaches its equilibrium state within a very short time as compared to the other gas species which may take many months of running.

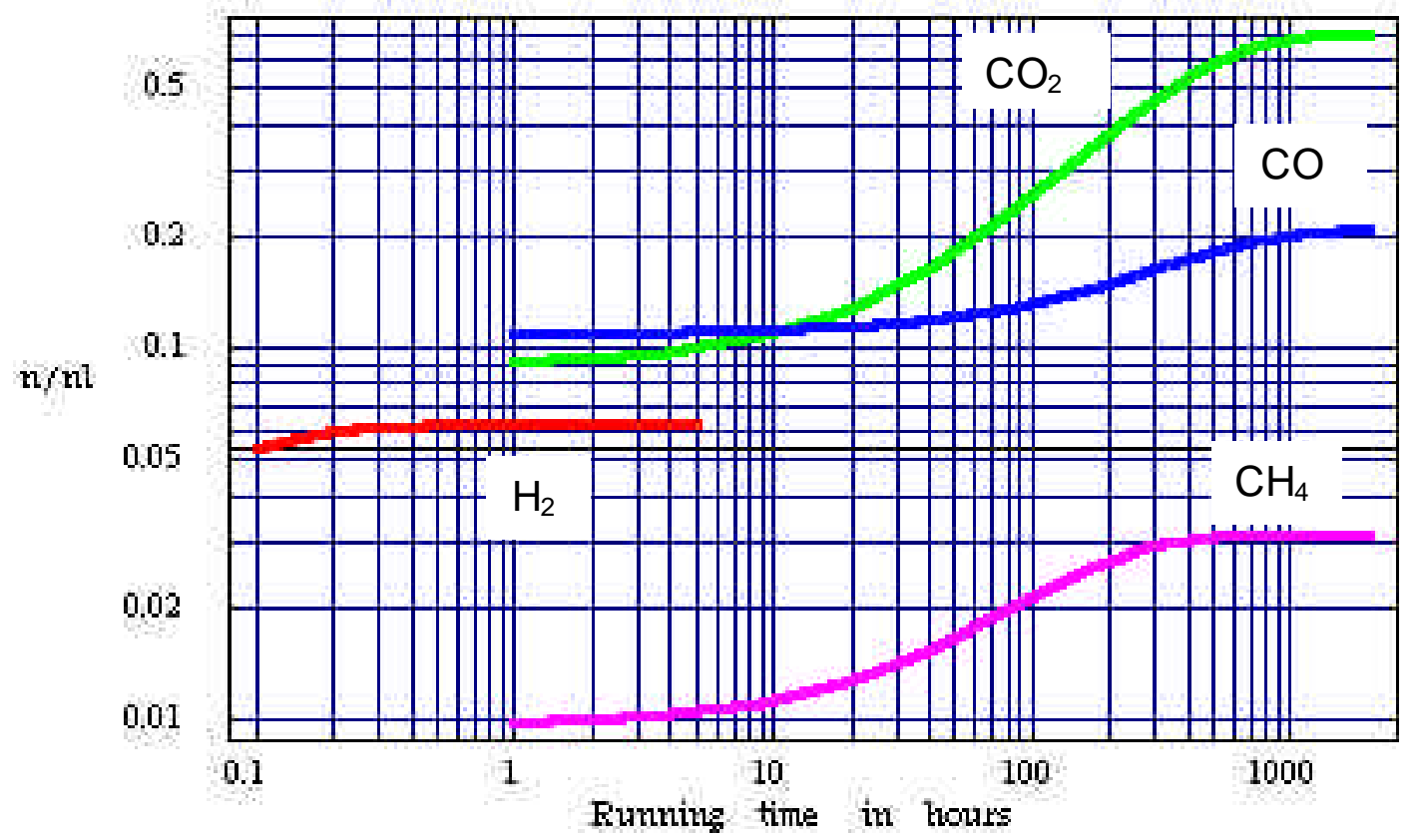

Fig. 4. Evolution of the gas density with LHC running time using the input data from Table 1. The gas densities have been normalised with respect to the values for a 100 hour beam lifetime $\left(n_{1}\right.$ for hydrogen $=10^{15} \mathrm{H}_{2} \cdot \mathrm{m}^{-3}$ ) .

A reduction of the PPY with photon dose is known from RT measurements and has also been observed at $77 \mathrm{~K}[5,6]$ but has not yet been confirmed below $20 \mathrm{~K}$. Concerning the dominant effect of $\mathrm{CO}_{2}$, there exist controversial results between experiments performed at CERN and at 
BINP in Novosibirsk which obtain a much lower desorption yield of $\mathrm{CO}_{2}$ at $5 \mathrm{~K}-10 \mathrm{~K}$. Due to the importance of its contribution to the global beam lifetime a smaller yield for $\mathrm{CO}_{2}$ would lead to a significant increase of the beam lifetime. Finally, it has been assumed that the PPY of $\mathrm{CO}_{2}$ and $\mathrm{CO}$ are constant between $114 \mathrm{~K}-20 \mathrm{~K}$ and $78 \mathrm{~K}-20 \mathrm{~K}$ respectively. But, it is known that the PPY decreases significantly in this temperature range $[2,5,6,8]$. The results for the gas species other than $\mathrm{H}_{2}$ should thus be taken as upper limits. Nevertheless, one may conclude that even with these pessimistic assumptions, the required vacuum performance of the LHC can be reached safely.

Fig. 5 shows the dynamic pressure evolution in the case of a pre-condensed gas layer on the inner wall of the BS. During a magnet quench, the gas load which has been accumulated on the $\mathrm{CB}$, e.g. during 1000 hours of operation, is desorbed during the warming up. Subsequently, in case the system would be cooled down in the wrong sequence so that the BS would be at a lower temperature than the $\mathrm{CB}$, an excessive amount will condense on the BS. The gas is assumed to be condensed in equal proportions on the inner and outer surface of the BS. The respective surface coverage of each gas species would be $25.110^{15} \mathrm{H}_{2} . \mathrm{cm}^{-2}, 0.7510^{15} \mathrm{CH}_{4} \cdot \mathrm{cm}^{-2}, 2.510^{15} \mathrm{CO} . \mathrm{cm}^{-2}$ and $4.410^{15} \mathrm{CO}_{2} \cdot \mathrm{cm}^{-2}$. During operation, the recycling of this excess amount of surface gas will lead to a transient pressure rise. Again, the redistribution of $\mathrm{H}_{2}$ occurs fast while the other species may take several weeks to reach their equilibrium. Minimisation of BS surface coverage will avoid undesirable pressure transient. The photocracking of $\mathrm{CH}_{4}$ and of $\mathrm{CO}_{2}$ molecules into $\mathrm{CO}$ and $\mathrm{H}_{2}$ respectively $[6,7]$ implies that the condensed $\mathrm{CO}_{2}$ would be transformed predominantly into $\mathrm{CO}$. The overall effect will be that the equivalent density remains below the curve shown in Fig. 5.

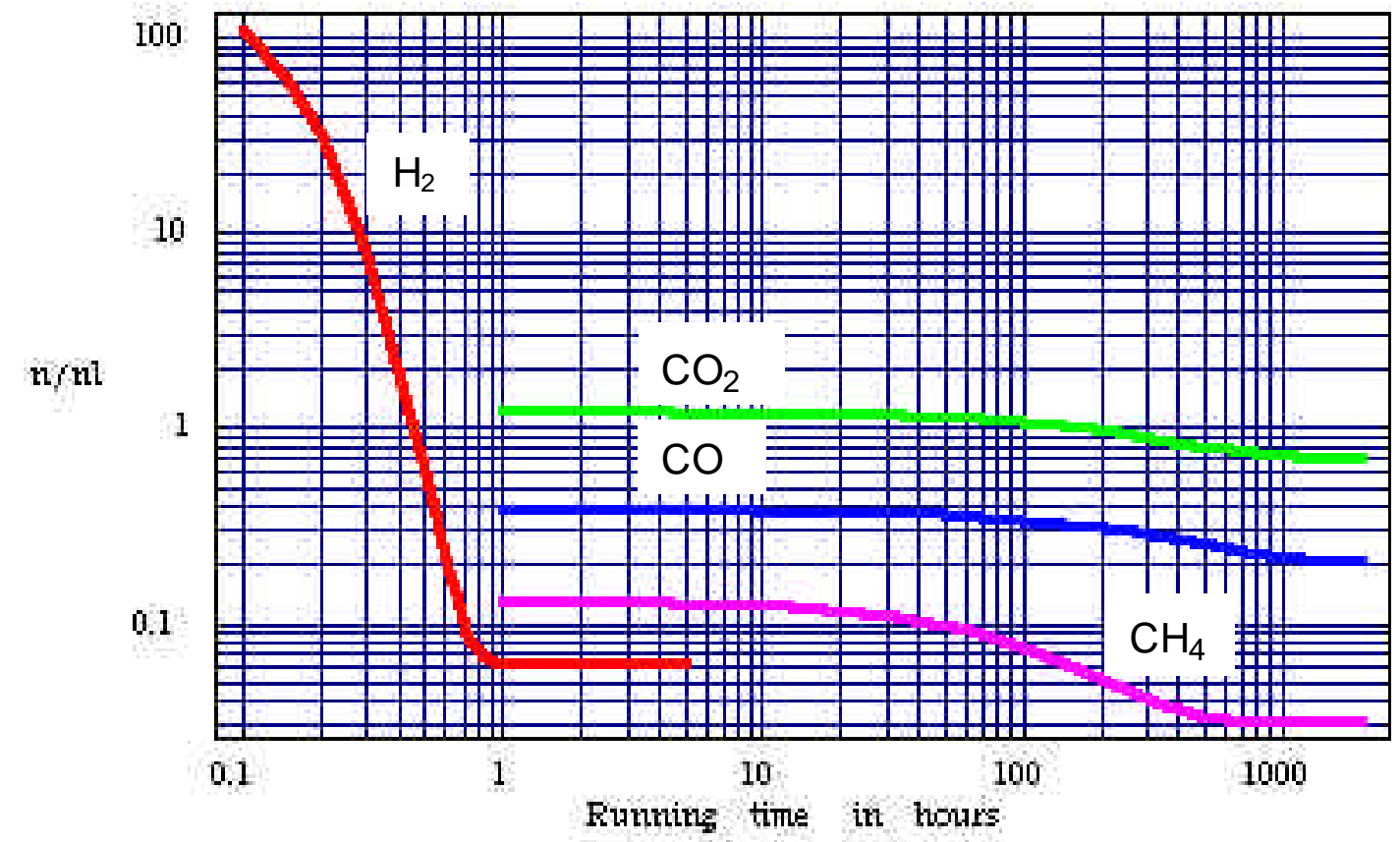

Fig. 5. Dynamic pressure evolution due to an excess amount of pre-condensed gas on the inner wall of the BS. The gas load is assumed to correspond to 1000 hours of operation.

\section{Conclusions}

PPY and the sticking coefficients as a function of temperature have been measured for an OFE copper BS subjected to SR. The photodesorbed gas species are $\mathrm{H}_{2}, \mathrm{CH}_{4}, \mathrm{CO}$ and $\mathrm{CO}_{2}$. For 
the purpose of the measurement method : a) care was taken to minimise the surface coverage on the BS and thus the recycling yield for each gas species and b) the PPY was assumed to be constant below the limiting temperature of thermal desorption $\mathrm{T}_{\mathrm{H}}$.

It is shown that the PPY decreases with decreasing temperature. For irradiation with SR of $194 \mathrm{eV}$ critical energy, the PPY of $\mathrm{H}_{2}$ below $20 \mathrm{~K}$ and after an accumulated dose of $3.510^{22}$ photons. $\mathrm{m}^{-1}$ is in the range $10^{-4}$ molecules.photon ${ }^{-1}$. The PPY of the other gases is in the range of $10^{-5}$ molecules.photon $^{-1}$. The sticking coefficient on a 'bare surface' decreases with increasing temperature. Below $20 \mathrm{~K}$, it is of the order of a few percent for all gases. At $15 \mathrm{~K}$ nominal LHC operation, the beam life time due to proton-nuclear scattering can be estimated as 100 hours even without beam conditioning. Regarding photon induced molecular desorption, a very modest cleaning of the BS by less than a factor of two will be sufficient to meet and even exceed the design goal for the LHC vacuum. To avoid undesirable pressure transients, the cool down of the BS should be controlled such that its temperature is higher than the $\mathrm{CB}$. Temporarily raising the BS temperature up to about $100 \mathrm{~K}$ should be considered as it would permit a fast transfer of gas from the $\mathrm{BS}$ to the $\mathrm{CB}$ prior beam operation.

\section{Acknowledgements}

The work performed by NIKHEF during COLDEX design and construction is gratefully acknowledged. We would like to thank Drs J-P. Potier and L. Rinolfi as well as the operating team for running the EPA machine with excellent beam quality. Discussions with Drs. V. Anashin, J. Gómez-Goñi, J-M. Laurent and O. Malyshev have greatly improved our understanding of the LHC cryogenic vacuum system.

\section{References}

[1] Gröbner O. Vacuum 60 (2001) 25-34.

[2] Anashin VV, Malyshev OB, Osipov VN, Maslennikov IL and Turner WC. J. Vac. Sci. Technol. A 12(5), 2917, Sep/Oct (1994).

[3] COLDEX : an experiment to simulate the LHC beam vacuum, paper in preparation.

[4] Benvenuti C, Calder RS and Passardi G. J. Vac. Sci. Technol. 13( 6), 1172 (1976).

[5] Calder R, Gröbner O, Mathewson AG, Anashin VV, Dranichnikov A and Malyshev OB. J. Vac. Sci. Technol. A 14(4), 2618, Jul/Aug (1996).

[6] Baglin V. Etude de la photo-désorption de surfaces techniques aux températures cryogéniques. Thèse, Université Denis Diderot Paris 7, May 1997.

[7] Anashin VV, Malyshev OB, Calder R, Gröbner O. Vacuum 53 (1999) 269-272.

[8] Baglin V. CERN, LHC Project Report 9, June 1996.

[9] Anashin VV, Malyshev OB, Calder R, Gröbner O and Mathewson AG. Vacuum 48/7-9/ (1997) 785-788. 